\title{
COMPOSIÇÃO DA SOLUÇÃO DE UM SOLO ALAGADO CONFORME A PROFUNDIDADE E O TEMPO DE ALAGAMENTO, UTILIZANDO NOVO MÉTODO DE COLETA(1)
}

\author{
R. O. SOUSA ${ }^{(2)}$, H. BOHNEN ${ }^{(3)} \&$ E. J . MEURER ${ }^{(4)}$
}

\begin{abstract}
RESUMO
Na coleta da solução de solos alagados, é importante evitar o contato do oxigênio com a solução para prevenir reações redox que podem modificar sua composição química. Neste trabalho, realizado em casa de vegetação, foram feitos estudos sobre o estado de oxirredução e sobre a composição química de um Planossolo, em diferentes profundidades e tempos de alagamento, visando propor um novo método para a coleta da solução de solos alagados. 0 estudo foi feito em vasos plásticos com $7 \mathrm{~kg}$ de material de solo proveniente da camada superficial $(0-20 \mathrm{~cm})$, num delineamento inteiramente casualizado, com três repetições. $\mathbf{O}$ dispositivo de coleta da solução do solo consistiu de um tubo de polieti leno com $70 \mathrm{~cm}$ de comprimento e $0,6 \mathrm{~cm}$ de diâmetro interno, com duas fileiras de orifíci os de $1 \mathrm{~mm}$ de diâmetro, recoberto com tela de nylon de 400 mesh, construído na forma de espiral e acomodado nas profundidades de 2,5 e $10 \mathrm{~cm}$. A este dispositivo foi acoplado um sifão de vidro para a coleta da solução. Após a instalação do sistema coletor, o solo foi mantido alagado por 109 dias, tendo sido feitas coletas semanais da solução nas três profundidades. Foram analisados os valores de $\mathrm{E}_{\mathrm{h}}$ e pH e os teores de $\mathrm{Mn}^{2+}, \mathrm{Fe}^{2+}, \mathrm{Ca}^{2+}, \mathrm{Mg}^{2+}$ e $\mathrm{K}^{+}$na solução. As condições de oxirredução e os teores de cátions na solução nas diferentes profundidades, durante o período em que o solo se manteve alagado, permitiram concluir que o método utilizado pode ser indicado para coleta da solução de solos alagados, embora haja necessidade de estudos mais aprofundados sobre o assunto.
\end{abstract}

Termos de indexação: oxirredução, solução do solo, alterações químicas.

\footnotetext{
(1) Trabalho apresentado no I Congresso Brasileiro de Arroz Irrigado e XXIII Reunião da Cultura do Arroz Irrigado, Pelotas, 2-5 de agosto de 1999. Recebido para publicação em dezembro de 2000 e aprovado em agosto de 2001.

(2) Doutorando em Ciência do Solo da Universidade Federal do Rio Grande do Sul - UFRGS e Professor Assistente do Departamento de Solos da Faculdade deAgronomia Eliseu Maciel da Universidade Federal de Pelotas - UFPEL. Caixa Postal 354, CEP 96001-970 Pelotas (RS). E-mail: rosousa@ufpel.tche.br

(3) Professor convidado do Departamento de Solos, UFRGS. Caixa Postal 776, CEP 90001-970 Porto Alegre (RS). E-mail: bohnen@conex.com.br

(4) Professor Adjunto do Departamento de Solos, UFRGS. Bolsista do CNPq. E-mail: egon.meurer@ufrgs.br
} 


\title{
SUMMARY: COMPOSITION OF A FLOODED SOIL SOLUTION AS AFFECTED BY DEPTH AND TIME OF FLOOD, USING A NEW COLLECTING METHODOLOGY
}

\begin{abstract}
When col lecting fl ooded soil solution, it is important to avoi d molecular oxygen contact to prevent redox reactions that can modify its chemi cal composition. This work, carried out in a greenhouse, analyzed the redox status and chemical composition of an Albaqualf, in different depths and duration of flooding, aiming to propose a new methodology to collect flooded soil solution. The study was conducted using $7.0 \mathrm{~kg}$ plastic pots, filled with soil material from the surface layer $(0-20 \mathrm{~cm})$ in a completely randomized design, with three replications. The col lecting device consisted of a spiral polyethylenetube $(70 \mathrm{~cm}$ long and $0.6 \mathrm{~cm}$ internal diameter) with small holes ( $1 \mathrm{~mm}$ diameter), covered with a nylon filter (400 mesh) and located in soil depths at 2, 5 and $10 \mathrm{~cm}$. A gl ass tube was connected to this deviceto coll lect thesol utions. Soil was maintained flooded during 109 days and soil solution samples were collected every week. The soil sol ution was analyzed for $\mathrm{Eh}, \mathrm{pH}, \mathrm{Mn}, \mathrm{Fe}, \mathrm{Ca}$, $\mathrm{Mg}$ and $\mathrm{K}$. Results of the redox status and the cation values in the solution, at different ti mes and depths, al lowed to concludethat this method can beused to study thefl ooded soil environment although further studies are necessary.
\end{abstract}

Index terms: oxyreduction, soil solution, chemical changes.

\section{INTRODUÇÃO}

A solução do sol oéo meio através do qual espécies químicas dissol vidas chegam atéà superfície da raiz e podem ser carregadas para águas subterrâneas ou superficiais. Está em contato com a fase sólida do solo e com outros componentes envolvidos na ciclagem biogeoquímica (Ritchie \& Sposito, 1995). A caracterização da solução do solo é importante para estudos de disponibilidade de nutrientes (fator intensidade), fluxos de elementos e compostos no perfil do solo, presença de el ementos tóxicos e estado de oxirredução do solo.

No entanto, a obtenção da solução do solo não é tarefa fácil, pois o método escolhido para tal deve possibilitar obter amostras com características físicoquímicas que representem a solução do solo e, se possível, ser de fácil execução. Diferentes métodos de coleta da solução do solo têm sido propostos (sucção, deslocamento, centrifugação, extrato saturado), apresentando diferentes aplicações e limitações, dependendo do objetivo e grau de exatidão exigido nos estudos (Adams, 1974; Suarez, 1999). Para o solo alagado, além disso, existe a necessi dade de limitar o contato com o oxigênio do ar atmosférico, pois este modifica o estado de oxirredução dos seus componentes, alterando a sua constituição química.

Procurando minimizar estes fatores, alguns pesquisadores utilizaram cápsulas porosas submetidas a vácuo (M oraes \& Dynia, 1990; Sousa, 1991), propostas na 1a Reunião sobre Ferro em Solos I nundados (Barbosa Filho, 1988) com o objetivo de uniformizar métodos. Foram identificados, porém, neste método, al guns fatores que podem ser fontes de erro, como: dificuldade em manter a solução do solo livre de oxigênio, uma vez que o sistema não é totalmente hermético; poros não-uniformes das cápsulas, que podem causar fluxo de solução diferenciado, resultando em maior ou menor risco de oxidação, dependendo do tempo de exposição da solução ao ar atmosférico; obstrução dos poros das cápsulas por colóides do sol o e preci pitação de óxidos/ hidróxidos de ferro na superfícieinterna da cápsula.

Alguns destes problemas foram minimizados por Vahl (1991), que utilizou outro método, sugerido por Bohnen (comunicação pessoal, 1990) com base na extração da solução por tubos plásticos perfurados e recobertos com tela de nylon extremamente fina.

Este trabal ho teve como objetivo estudar as variações nas condições de oxirredução e composição da solução, col etada em diferentes profundidades de um solo alagado, visando propor novo método de coleta.

\section{MATERIAL E MÉTODOS}

No experimento, realizado em casa de vegetação, localizada na Faculdade de Agronomia da UFRGS, utilizou-se material da camada de $0-20 \mathrm{~cm}$ de profundidade de um Planossolo do município do Capão do Leão (RS). As análises do solo, efetuadas segundo método descrito em Tedesco et al. (1995), indicaram os seguintes resultados: $\mathrm{pH}$ (água) $=5,2$; $13 \mathrm{mg} \mathrm{L}^{-1}$ de P; $36 \mathrm{mg} \mathrm{L}^{-1}$ de K ; 3,0 $\mathrm{cmol}_{\mathrm{C}} \mathrm{L}^{-1}$ de Ca; $1,8 \mathrm{cmol}_{\mathrm{C}} \mathrm{L}^{-1}$ de $\mathrm{Mg}$; $17 \mathrm{~g} \mathrm{~kg}^{-1}$ de matéria orgânica; 
1,4 $\mathrm{g} \mathrm{kg}^{-1}$ de Fe extraído com oxalato de amônio a $\mathrm{pH}$ 3; e $160 \mathrm{~g} \mathrm{~kg}^{-1}$ de argila. O delineamento experimental adotado foi o inteiramente casual izado, com três repetições.

O sistema empregado para coletar a solução do solo consistiu de um tubo de polietileno com $70 \mathrm{~cm}$ de comprimento, $0,6 \mathrm{~cm}$ de diâmetro interno e $0,7 \mathrm{~cm}$ de diâmetro externo perfurado com duas fileiras de pequenos orifícios com aproximadamente $1 \mathrm{~mm}$ de diâmetro. Em uma das extremidades do tubo, com cerca de $5 \mathrm{~cm}$ não perfurada, conectou-se um tubo de silicone com $5 \mathrm{~cm}$ de comprimento. A outra extremidade foi fechada com ferro aquecido. O tubo de polietileno foi revestido com tela de nylon com malha de 400 mesh ao longo de toda porção perfurada, amarrando-se firmemente com linha de nylon, para impedir que partículas mais finas do solo penetrassem no tubo junto com a solução do sol o. O tubo foi enrolado sobre si, na forma de uma espiral (serpentina), mantendo-se aproximadamente $2 \mathrm{~cm}$ de distância entre cada volta da espiral. A espiral foi conectada a um tubo de vidro, na forma de sifão, projetado para fora do vaso, para permitir a retirada da solução do solo sem utilizar sucção forte, o que poderia causar entupimento da tela de nylon ou arrastar partículas de sol o junto com a solução. $\mathrm{Na}$ extremidade livre do sifão, foi colocado um tubo de silicone com $2 \mathrm{~cm}$ de comprimento, fechado com um tampão de vidro.

O solo utilizado no experimento foi passado em peneira com malha de $10 \mathrm{~mm}$ e colocado em vasos plásticos, na quantidade de $7 \mathrm{~kg}$ de sol o seco por vaso. Os dispositivos para a coleta da solução foram acomodados nas profundidades de 2, 5 e $10 \mathrm{~cm}$. O sistema foi mantido com umidade próxima à capacidade de campo por um período de 10 dias, após o qual se iniciou o alagamento, mantendo-se uma lâmina deágua com cerca de $5 \mathrm{~cm}$ dealtura durante 109 dias. Nesse período, foram feitas coletas semanais da sol ução para o estudo das condições de oxirredução e composição química.

As análises de $\mathrm{pH}$ e $\mathrm{E}_{\mathrm{h}}$ da solução do solo foram feitas por meio de el etrodos específicos, combinados, ligados a um potenciômetro e previamente instalados em uma "célula el etrométrica", construída em resina de vidro e acrílica (Figura 1). Essa célula dispunha de um pequeno tubo de entrada na parte inferior, que, na amostragem, foi conectado diretamente à extremidade do sifão de coleta da solução. A parte superior da célula apresentava outro tubo, por onde era expeli do o excesso de solução do solo. Como a célula permanecia cheia de solução durante as leituras, foi possível minimizar o contato desta com o oxigênio atmosférico, diminuindo os riscos de alteração das suas características eletroquímicas. A pós as determinações el etroquímicas, transferiram-se aproximadamente $10 \mathrm{~mL}$ de solução da célula diretamente para frascos de vidro, onde havia sido colocado previamente $1 \mathrm{~mL}$ de $\mathrm{HCl}$

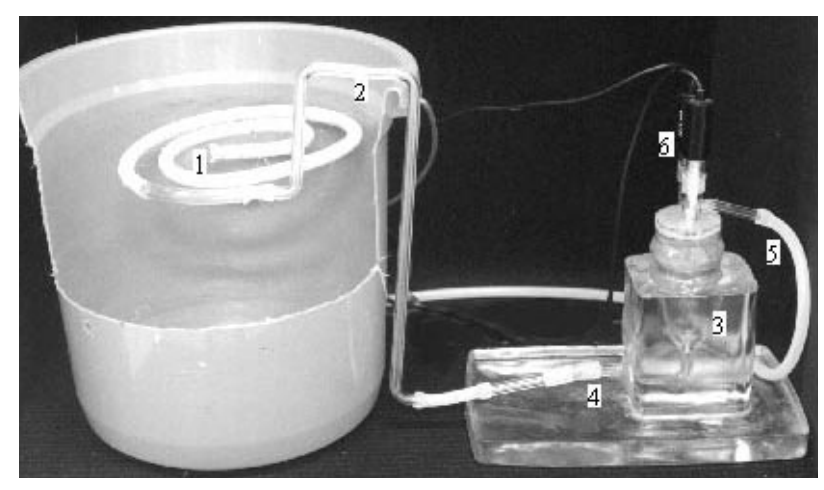

Figura 1. Sistema de extração da solução do solo acoplado à célula eletrométrica de determinação do potencial redox e pH. ${ }^{1}$ Tubo de polietileno perfurado e recoberto com tela de nylon; ${ }^{2}$ Tubo de vidro em forma de sifão; ${ }^{3}$ Câmara eletrométrica; ${ }^{4}$ Tubo de entrada e saída da solução; ${ }^{5}$ Tubo de descarga do excesso de solução; ${ }^{6}$ Eletrodos de medida do E h e pH.

$1,1 \mathrm{~mol} \mathrm{~L}-1$, para quea concentração final na amostra ficasse em torno de $0,1 \mathrm{~mol} \mathrm{~L}^{-1}$. A diluição da amostra foi calculada por diferença de pesagem, antes e depois da col ocação do ácido eda amostra no frasco de vidro. Nesta amostra, foram avaliados os cátions por espectroscopia de absorção atômica $\left(\mathrm{Mn}^{2+}, \mathrm{Fe}^{2+}, \mathrm{Ca}^{2+} \mathrm{eMg}^{2+}\right)$ efotometria de chama $(\mathrm{K}+)$.

Os valores de $E_{h}$ foram corrigidos para o potencial padrão do el etrodo de referência (calomelano), conforme a fórmula: $\mathrm{E}_{\mathrm{h}}=\mathrm{E}_{\text {medido }}+248 \mathrm{mV}$, e, a partir dos valores de Eh corrigidos, foi calculado o pe por: $\mathrm{pe}=\mathrm{E}_{\mathrm{h}} / 59$. Dos val ores de $\mathrm{pH}$ e $\mathrm{E}_{\mathrm{h}}$ medidos, calculouse o parâmetro redox pe $+\mathrm{pH}$.

A eficiência do método empregado foi avaliada por meio da interpretação dos resultados da análise química da solução col etada nas diferentes profundidades, conforme descrição prévia, bem como por comparação com outros métodos citados na literatura.

\section{RESULTADOS E DISCUSSÃO}

$\mathrm{O}$ pe $+\mathrm{pH}$ diminuiu com o alagamento, estabilizando-sea partir dos 28 dias (F igura 2). Esse comportamento é característico de um solo alagado e ocorre em decorrência da redução de compostos oxidados pela atividade dos microrganismos anaeróbios, que utilizam essas substâncias como receptores de elétrons nas rotas bioquímicas de produção de energia. Observa-se que o pe $+\mathrm{pH}$ diminuiu em profundidade, pois o oxigênio dissol vido na lâmina de água pode difundir por alguns 
centímetros no solo (Shu-Zheng, 1985), mantendo um ambientemais oxidado na superfície. Resultados semel hantes foram obtidos por Madruga (1999), na solução de um Planossolo alagado, onde constatou, a partir dos 36 dias de alagamento, que o valor de $\mathrm{pe}+\mathrm{pH}$ diminuiu de 12 para oito, estabilizando-se neste valor.

As concentrações de $\mathrm{Mn}^{2+}$ e $\mathrm{Fe}^{2+}$ na solução do solo (Figuras 3 e 4) aumentaram durante o alagamento até atingirem um pico aos 35 dias, diminuindo gradativamente para valores que se mantiveram estáveis. O aumento na concentração destes elementos deveu-se à redução dos óxidos mangânicos e férricos e à posterior diminuição decorrente do aumento do $\mathrm{pH}$ que diminuiu a solubilidade desses minerais (Ponnamperuma, 1972).

Os teores de Mn e Fe na solução do solo foram maiores a $10 \mathrm{~cm}$ de profundidade, visto que o oxigênio molecular próximo à superfície do solo, difundido lentamente através da lâmina de água, limita a redução dos óxidos de manganês e ferro. A variação nos teores de ferro na solução do solo foi semel hante à observada por outros autores (Dias, 1991; Madruga, 1999; Silva et al., 2000) em Planossol os alagados. Nesses trabal hos, também foi observado aumento nos teores de ferro na solução até 45 dias de alagamento, atingindo val ores médios de $200 \mathrm{mg} \mathrm{L}^{-1}$, diminuindo para concentrações médias de $130 \mathrm{mg} \mathrm{L}^{-1}$ que se mantiveram constantes até o final do período de alagamento.

Houve um aumento na concentração de $\mathrm{Ca}^{2+} \mathrm{e}$ $\mathrm{Mg}^{2+}$ na solução (Figuras 5 e 6) que atingiram um valor máximo aos 35 dias de alagamento, decrescendo, após, para val ores que se mantiveram aproximadamente constantes até o final do período

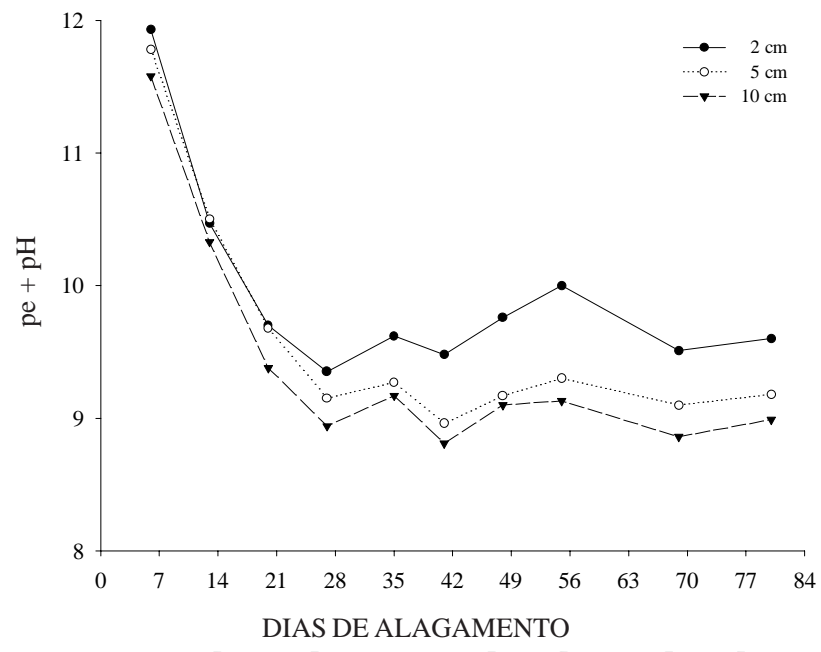

Figura 2. Valores de pe + pH da solução do solo em três profundidades de um P lanossolo submetido a alagamento.

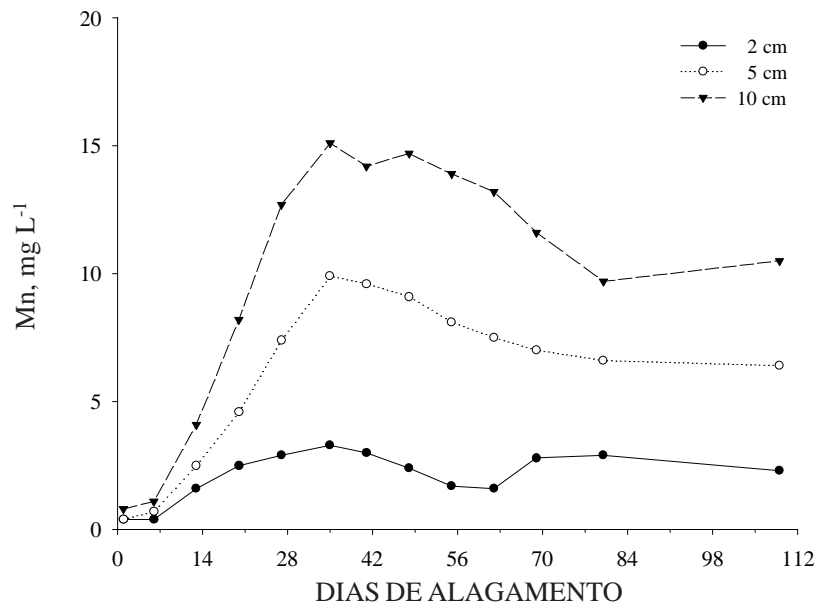

Figura 3. Concentração de manganês na solução do solo em três profundidades de um Planossolo submetido a alagamento.

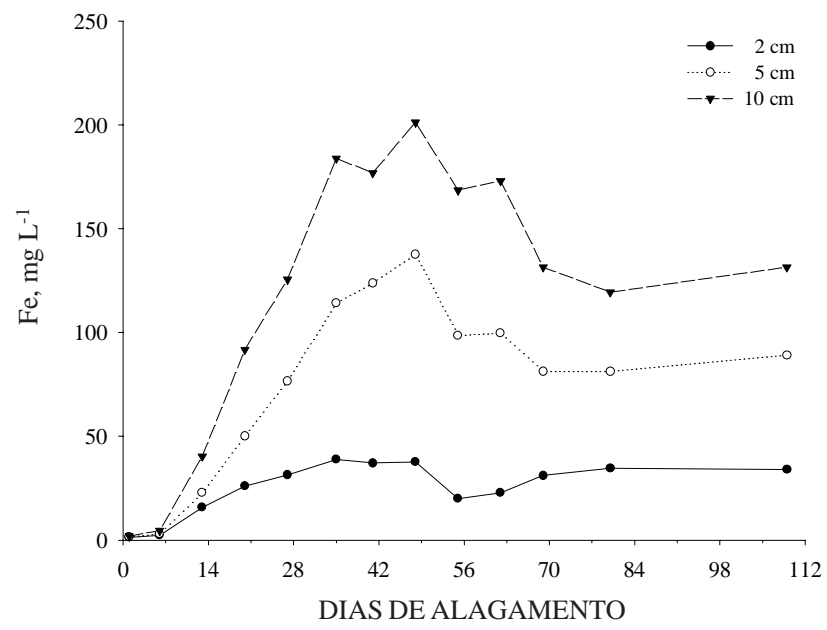

Figura 4. Concentração de ferro na solução do solo em três profundidades de um Planossolo submetido a alagamento.

deamostragem. Os teores de $\mathrm{Ca}^{2+} \mathrm{eMg}^{2+}$ aumentaram em profundidade, semelhantemente ao observado para o $\mathrm{Fe}^{2+}$ e o $\mathrm{Mn}^{2+}$, provavel mente deslocados dos sítios de troca pel o $\mathrm{Fe}^{2+}$, principalmente, epelo $\mathrm{Mn}^{2+}$.

Os teores de K+ na solução do solo apresentaram variação durante o período de al agamento entre as profundidades amostradas. Os teores de $\mathrm{K}^{+}$em solução foram muito baixos e as diferenças observadas revel aram oscilações normais (Figura 7).

Analisando os resultados obtidos (Figuras 2, 3, 4, 5, 6 e 7), verificou-se a coerência entre eles e o ambiente de sol o reduzido. A diminuição do pe $+\mathrm{pH}$ foi acompanhada pelo aumento dos teores de ferroe manganês, e a cinética de cálcio e magnésio acompanhou aquelas do ferro e do manganês. As 


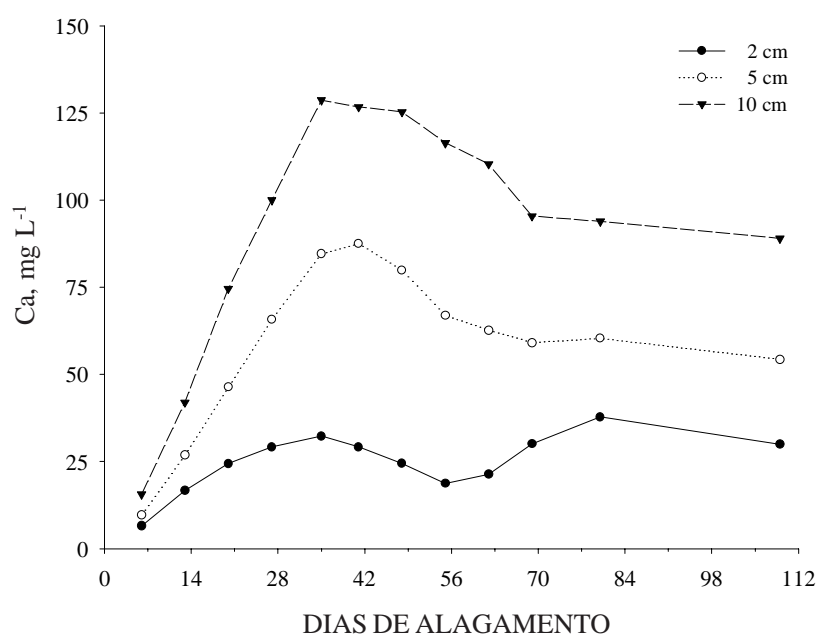

Figura 5. Concentração de cálcio na solução do solo em três profundidades de um Planossolo submetido a alagamento.

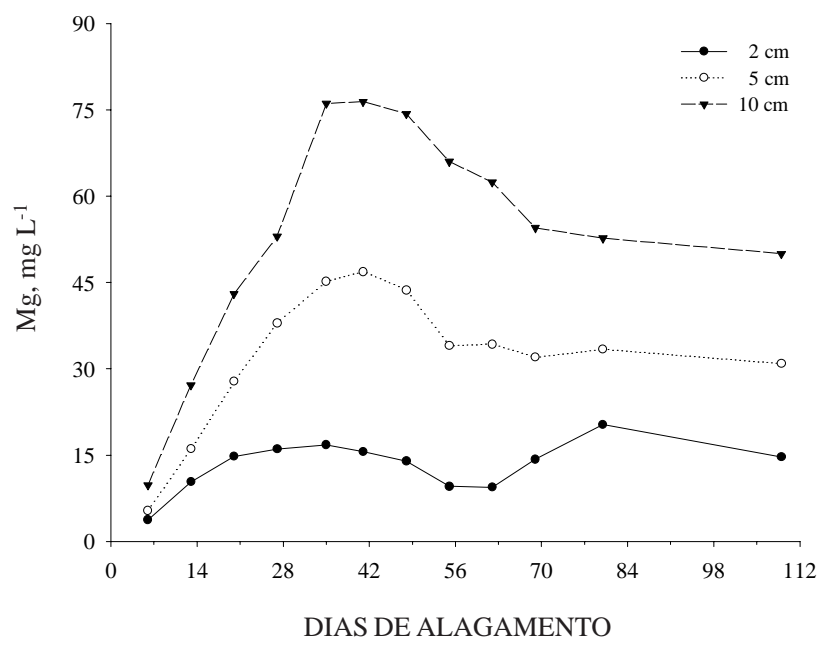

Figura 6. Concentração de mangnésio na solução do solo em três profundidades de um Planossolo submetido a alagamento.

condições mais reduzidas na profundidade de $10 \mathrm{~cm}$, evidenciadas pel o parâmetro redox, são confirmadas pelas maiores concentrações de manganês, ferro, cál cio e magnésio. A concordância entre os indicadores foi evidenciada até em variações mais pontuais: por exemplo, o aumento do pe $+\mathrm{pH}$ observado a $2 \mathrm{~cm}$ de profundidade, aos 56 dias de alagamento, foi acompanhado pela diminuição nos teores dos elementos avaliados.

O parâmetro redox e os teores de cátions em solução foram semel hantes aos observados por outros autores que também estudaram estes indicadores na solução de Planossolos (Dias, 1991; Madruga, 1999; Silva et al., 2000). Tal comportamento reforça a idéia de que os dados deste trabalho são

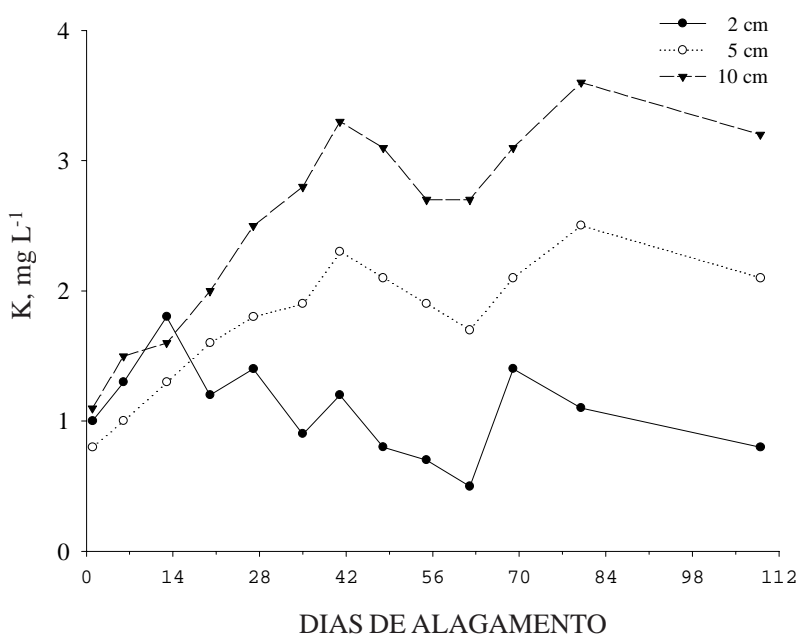

Figura 7. Concentração de potássio na solução do solo em três profundidades de um Planossolo submetido a alagamento.

condizentes com o ambiente de sol o reduzido, e que o método empregado foi eficaz para discriminar as alterações eletroquímicas e químicas ocorridas durante $o$ alagamento.

Nas primeiras col etas, foi observada sedimentação de pequena quantidade de partículas finas de solo, no fundo dos frascos de coleta, que diminuiu nas extrações subseqüentes. Este material pode conter cáti ons adsorvidos, desl ocados para a solução do solo quando esta é acidificada. Testes realizados com amostras filtradas e não filtradas indicaram não haver diferenças nos indicadores avaliados, neste caso (dados não apresentados), provavel mente por ter sido a quantidade de material depositado pequena. Nãoé possível garantir queisto não venha a ser problema para outros experimentos, razão por queéaconsel hável que se façam testes com amostras filtradas e não filtradas para maior segurança.

Observou-se que ofluxo de solução foi diminuindo ao longo das col etas, indicando que os poros da tela de nylon foram sendo obstruídos aos poucos, por partículas de solo. Em solos argilosos ou com alta quantidade de argila dispersa, este problema pode ser agravado, pois, com a diminuição do fluxo, há necessidade de maior tempo para recol her a solução na célula eletroquímica, aumentando o risco de oxidação, o que pode causar erros, principalmente nas medidas de potencial redox.

\section{CONCLUSÕES E RECOMENDAÇÕES}

1. As variações no estado de oxirredução e nos teores de cátions trocáveis na solução, considerando as profundidades e o tempo de alagamento, revelaram que o uso de tubos de polietileno 
perfurados e revestidos com tela de nylon de 400 mesh, mostrou-se eficiente para a coleta da solução em solos alagados.

2. Há necessidade de estudos mais aprofundados para a proposição de um método que sirva de referência para a col eta da sol ução em sol os al agados.

3. O dispositivoe o método propostos para a col eta da solução de solo alagado foram eficazes para discriminar as modificações dos teores dos indicadores utilizados de acordo com a profundidade de amostragem e o tempo de alagamento.

\section{LITE RATURA CITADA}

ADAMS, F. Soil solution. In: CARSON, E.W., ed. The plant root and its environment. Charlottesville, University of Virginia Press, 1974. p. 441-481.

BARBOSA FILHO, M.P., coord. REUNIÃO SOBRE FERRO EM SOLOS INUNDADOS, 1., Goiânia, 1987. Anais. Goiânia, Empresa Brasileira de Pesquisa Agropecuária, 1988. 205p.

DIAS, A.D. Caracterização de parâmetros de solo aerado que permitam prever a ocorrência de toxidez por ferro em arroz irrigado. Pelotas, Universidade Federal de Pelotas, 1991. 65p. (Tese de Mestrado)

MADRUGA, E.F. Efeito da aplicação de resíduo vegetal e nitrato sobre a redução do solo. Pelotas, Universidade Federal de Pelotas, 1999. 47p. (Tese de Mestrado)
MORAES, J.F.V. \& DYNIA, J.F. Uso de cápsulas porosas para extrair solução do solo. Pesq. Agropec. Bras., 25:1523$1528,1990$.

PONNAMPERUMA, F.N. Thechemistry of submerged soils. Adv. Agron., 24:29-96, 1972.

RITCHIE, G.S.P. \& SPOSITO, G. Speciation in Soils. In: URE, A.M. \& DAVIDSON, C.M., eds. Chemical speciation in the environment. Glasgow, Blackie Academic \& Professional, 1995. p.201-233.

SHU-ZHENG, P. Oxygen. In: TIAN-REN, Y., ed. Physical chemistry of paddy soils. Hong Kong, $C \& C$ J oint Printing Co., 1985. p.47-68.

SILVA, L.S.; SOUSA, R.O.; BOHNEN, H. Dinâmica de ferro e de potássio na solução de solo alagado em diferentes profundidades, na presença e na ausência de plantas de arroz. In: REUNIÃO SUL-BRASILEIRA DE CIÊNCIA DO SOLO, 3., Pelotas, 2000. Resumos Expandidos. Pelotas, Núdleo Regional Sul da SBCS, 2000. (CD-ROM).

SOUSA, R.O. Alternativas tecnológicas que visam minimizar o efeito da toxidez por ferro em arroz irrigado (Oryza sativa L.). Pelotas, Universidade Federal de Pelotas, 1991. 96p. (Tese de Mestrado)

SUAREZ, D.L. Thermodinamics of the soil solution. In: SPARKS, D.L., ed. Soil physical chemistry. 2.ed. Boca Raton, CRC Press, 1999. p.97-134.

TEDESCO, M.J .; GIANELLO, C.; BISSANI, C.A.; BOHNEN, H. \& VOLKWEISS, S.J. Análises de solo, plantas e outros materiais. 2.ed. Porto Alegre, Universidade Federal do Rio Grande do Sul, 1995. 174p. (Boletim Técnico, 5)

VAHL, L.C. Toxidez de ferro em genótipos de arroz irrigado por alagamento. Porto Alegre, Universidade Federal do Rio Grande do Sul, 1991. 167p. (Tese de Doutorado) 